\title{
The new Göttingen Fabry-Pérot spectrometer for two-dimensional observations of the Sun
}

\author{
K. G. Puschmann ${ }^{1}$, F. Kneer ${ }^{1}$, T. Seelemann ${ }^{1,2}$, and A. D. Wittmann ${ }^{1}$ \\ 1 Institut für Astrophysik, Friedrich-Hund-Platz 1, 37077 Göttingen, Germany \\ e-mail: kgp@astro.physik.uni-goettingen . de \\ ${ }^{2}$ LaVision GmbH, Anna-Vandenhoeck-Ring 19, 37081 Göttingen, Germany
}

Received 7 November 2005 / Accepted 10 March 2006

\section{ABSTRACT}

\begin{abstract}
Studies of small-scale dynamics and magnetic fields in the solar atmosphere require spectroscopy and polarimetry with high spatial resolution. For this purpose, spectrometers based on Fabry-Pérot interferometers (FPIs) have advantages over slit spectrographs. They possess a high throughput and allow fast two-dimensional, narrow-band imaging and image reconstruction of the data. In the present contribution we describe an upgrade, essentially renewal, of the Göttingen FPI spectrometer achieved during the first half of 2005. A new etalon from IC Optical Systems Ltd. (formerly Queensgate), England, with $70 \mathrm{~mm}$ free aperture for high spectral resolution has been mounted. New CCD detectors from LaVision GmbH (Göttingen) with powerful computer hard- and software were implemented. We consider the product of signal-to-noise ratio, frame rate, and field of view as a measure of the efficiency. At low light levels, e.g. in narrow-band speckle applications, this product has increased by a factor $\sim 60$ compared to the old system. In addition, several spectral regions can now be scanned quasi-simultaneously. We present first results obtained with the upgraded spectrometer. The efforts are undertaken to provide an up-to-date post-focus instrument for the new German $1.5 \mathrm{~m}$ GREGOR solar telescope presently under construction at the Observatorio del Teide on Tenerife.
\end{abstract}

Key words. instrumentation: high angular resolution - techniques: high angular resolution - techniques: image processing techniques: spectroscopic

\section{Introduction}

For the understanding of the fine-structure of the solar atmosphere and of its dynamics two-dimensional spectroscopic and polarimetric observations at high spatial, spectral and temporal resolution are required. Fabry-Pérot interferometers (FPIs) allow efficient narrow-band imaging in two dimensions. They are widely in use in astronomical spectroscopic observations. Their advantage compared to slit spectrographs lies in the twodimensional (2D) field of view (FOV) and high transmission, while, admittedly, their spectral coverage is limited. Here only references to some solar facilities are given: one of the first 2D FPI spectrometers was built for the Australian solar facility in Narrabri with three etalons (Ramsay et al. 1970; Loughhead et al. 1978; Bray 1988). The Arcetri group (Bonaccini et al. 1989) developed a spectrometer with an FPI in tandem with a Universal Birefringent Filter (UBF). Its further development is in use as the Italian Panoramic Monochromator (IPM, Cavallini 1998) at the French-Italian solar telescope THEMIS on Tenerife. A two-etalons spectrometer, the Interferometric BIdimensional Spectrometer (IBIS) from the Arcetri group (e.g. Cavallini et al. 2000; Cavallini 2002; Cavallini et al. 2003) is operative since June 2003 at the Dunn Solar Telescope of the National Solar Observatory (USA-NM). For the balloon-borne (later in space) SUNRISE solar telescope the Imaging MAgnetograph eXperiment, IMAX (Martínez Pillet et al. 2004) with one etalon used in double-pass is under construction. Since 1998 the Freiburg group is operating the TESOS instrument (Triple Etalon SOlar Spectrometer) in the German Vacuum Tower Telescope (VTT) at the Observatorio del Teide (Kentischer et al. 1998; von der Lühe \& Kentischer 2000; Tritschler et al. 2002)
The Universitäts-Sternwarte Göttingen (now Institut für Astrophysik) developed a 2D spectrometer for the VTT starting 1986 and presented first results obtained with it in the early 1990s (Bendlin et al. 1992; Bendlin 1993; Bendlin \& Volkmer 1993, 1995). Polarimetry with this 2D spectrometer was performed by Volkmer et al. (1995), among others, using a Stokes $V$ polarimeter.

From the beginning of the development of the Göttingen FPI we aimed at good spectral resolution and polarimetric sensitivity, and best spatial resolution in 2D fields of view (FOVs). In its first setup, the Göttingen FPI spectropolarimeter worked with a UBF as a pre-filter for order sorting for one narrow-band FPI, similarly as the IPM. The UBF was mounted in a telecentric position near a focus of the telescope, while the FPI was always mounted in the collimated beam near an image of the telescope pupil (see Kneer \& Hirzberger 2001 for a discussion of telecentric vs. collimated mounting). Since the UBF exhibited a low transmission, of the order of $10 \%$ or less, the exposure times were rather long, approximately $100 \mathrm{~ms}$. This ruled out application of speckle methods for image reconstruction.

The UBF was replaced in 2000 by an etalon with $0.125 \mathrm{~mm}$ spacing from Queensgate, and this etalon was mounted also in the collimated beam. This led to a reduction of the exposure times to $30 \mathrm{~ms}$ and less. The spectrometer was successfully used, since then, with post factum image reconstruction, for 2D spectroscopy and polarimetry with high spatial resolution (e.g. Koschinsky et al. 2001; Wunnenberg et al. 2002; Janßen et al. 2003; Okunev \& Kneer 2004; Bello González et al. 2005, and further references in these publications).

We describe in the following an upgrade, essentially renewal, accomplished in 2005 , which resulted in a substantial 


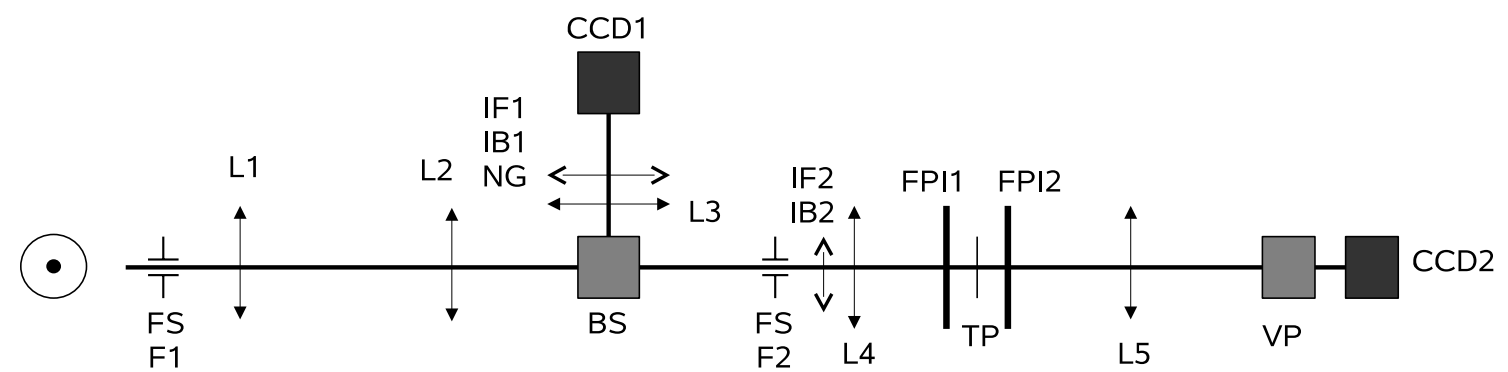

Fig. 1. Scheme of present optical setup of the spectrometer with FPIs in image of pupil, i.e. in collimated beam. F1: focus of the VTT after the Adaptive Optics system; L1 and L2: achromatic lenses with $f=1800 \mathrm{~mm}$ each to transfer the focus into an optical laboratory of the VTT; BS: beam-splitting cube; L3: achromat, reduces the focal length of the telescope by a factor 1.89; IF1, IB1, and NG: broadband interference filter, infrared blocking filter, and neutral density filter, respectively; CCD1: broadband CCD; F2: focus at the entrance of the spectrometer; FS: field stops at F1 and F2; IB2 and IF2: infrared blocking filter and narrow-band (FWHM 6-10 $)$ interference filter, respectively; L4: achromatic lens with $f=2250 \mathrm{~mm}$; FPI1 and FPI2: Fabry-Pérot interferometers at image of telescope pupil (TP); L5: achromatic lens with $f=1190 \mathrm{~mm}$; VP: polarimeter, e.g. Stokes $V$; CCD2: narrow-band CCD.

improvement of the performance of the spectrometer. We present details of the FPIs, new CCD detectors, and the new hard- and software for FPI control and data acquisition. Then we discuss the issue of unequal wavelength scanning by two different etalons. We give examples of first results obtained with the upgraded, essentially new, spectrometer and finally, we summarise its performance.

\section{Spectrometer upgrades}

Figure 1 shows the present optical setup of the spectrometer at the VTT. Two achromatic lenses (L1, L2) transfer the science focus following the Adaptive Optics system (KAOS, Soltau et al. 2002; von der Lühe et al. 2003) to an optical laboratory. A beam splitting cube feeds $5 \%$ of the incoming light into the broadband channel, where images are taken by the CCD1 detector using an interference filter $(F W H M \sim 100 \AA)$, an infrared blocking filter and a neutral density filter, respectively.

95\% of the incoming light is transmitted into the narrowband channel, where the light beam passes a narrow-band interference filter ( FWHM 6-10 А) and another infrared blocking filter. The achromatic collimator lens L4 produces an image of the telescope pupil where in its vicinity the two Fabry-Pérot etalons are mounted. A synchronised change of the spacing of the plates of these two etalons has the effect of a tunable filter which is used to scan along wavelength. FPI1 has a high reflectivity coating (reflectivity $R \sim 0.95$ ) in the wavelength range 5300-8600 where it gives a narrow transmission with $F W H M=28-75 \mathrm{~m} \AA$ which defines the spectral resolution of the spectrometer. The transmission of FPI2 is broader and suppresses the other orders of FPI1. The above mentioned narrow-band interference filter is used to select one of the orders of FPI2. The camera lens L5 produces a focus at the CCD2 detector where images are taken simultaneously with the broadband images. For polarimetry a Stokes $V$ polarimeter can be placed in front of CCD2. By binning pixels of the new CCDs $2 \times 2$ and replacing the former L5 with a focal length of $2250 \mathrm{~mm}$ by a lens with a focal length of $1190 \mathrm{~mm}$ a pixel scale of $0.112 /$ pix is achieved, comparable with $0.105 /$ pix of the old system. In the broadband channel an additional lens L3 is used to reduce the focal length of the telescope by a factor 1.89 to provide the same image scale as in the narrow-band channel.

The field stop at F1 limits the total amount of light entering the spectrometer and is larger than the FOV. The stop at F2 can be adjusted to the final FOV on the CCD2 detector. It allows to block the inter-etalon reflexes, which are subsequently removed from the beam by tilting one of the etalons (see Sect. 2.5 below), and to avoid overlapping of images separated by the $\checkmark$ polarimeter.

\subsection{New CCD system}

The old CCDs with Thomson TH 7863 FT chips, $384 \times 286$ pixel, have been replaced by two Imager QE CCDs from LaVision GmbH (Göttingen). The new CCDs have the Sony chip ICX285AL. This chip is a progressive scan type with electronical shutter. Each pixel element holds an image (light sensitive) area and a memory (covered) area. The chip is equipped with a matrix of micro-lenses that focus the light onto the image area of each pixel. The open and closing time of the electronical shutter is less than $1 \mu \mathrm{s}$. The CCDs have a full well capacity of $18000 \mathrm{e}^{-}$ and a read-out noise of $4.5 \mathrm{e}^{-}$. The $\mathrm{AD}$ conversion is done with 12 bit resolution. The CCD electronics allow three different operation modes: 1) Gain low: $\mathrm{AD}$ conversion factor $=4$ electrons to cover almost the full well capacity of the chip with 12 bit, 2) gain high: $\mathrm{AD}$ conversion factor $=2$ electrons to cover almost half of the full well capacity with 12 bit, and 3) high QE. Within this high $\mathrm{QE}$ mode the $\mathrm{AD}$ conversion factor is also 2 electrons. Additionally the chip is operated with higher substrate voltage which increases the quantum efficiency of the chip at higher wavelengths. The detectors have a spectral range from $3200 \AA$ up to $9000 \AA$ with a maximum quantum efficiency of $60 \%$ at $5500 \AA$. When observations in the infrared at $8600 \AA$ are to be performed the quantum efficiency amounts to $16 \%$. See Fig. 2 for the sensitivity curve of the new CCDs.

The chip has a size of $8.8 \times 6.7 \mathrm{~mm}^{2}$ which corresponds to $1376 \times 1040$ pixels with a pixel size of $6.45 \times 6.45 \mu \mathrm{m}^{2}$. In the actual setup a binning of $2 \times 2$ pixels is used corresponding to $688 \times 520$ pixels, yielding a total FOV of $77^{\prime \prime} \times 58^{\prime \prime}$ and an image scale of $0.112 /$ pixel.

\subsection{Programmable time unit - PTU}

The cameras are triggered and synchronised by a programmable timing unit (PTU) with an internal rate generation. The cameras take permanently images with a certain fixed frequency without interrupt to insure equal conditions during the whole observing run. The storage of the required images is controlled by software. The data are directly written onto a raid system of 4 hard disks thus providing $600 \mathrm{~GB}$ of direct storage capacity. The cameras 


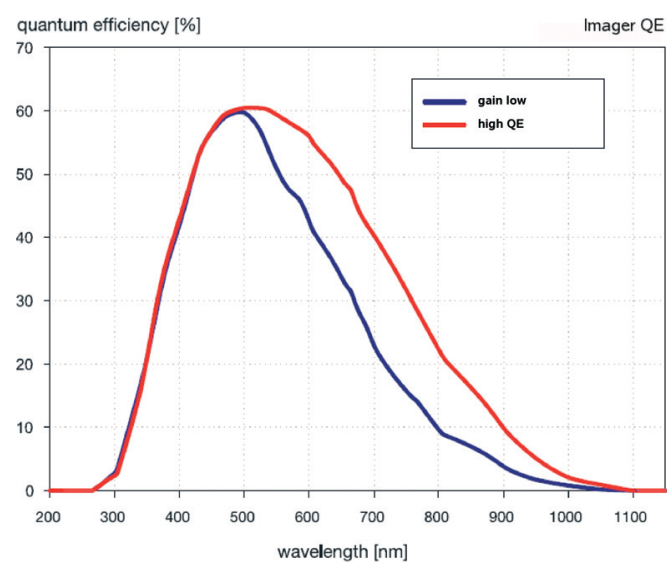

Fig. 2. Sensitivity curve of the CCDs. See text for the explanation of gain low and high $Q E$ mode.

do only accept external triggers, if the previous image acquisition is finished. The maximum frame rate $\left(f_{\mathrm{b}}\right)$ depends on exposure time $\left(t_{\text {exp }}\right)$, read-out time $\left(t_{\text {ro }}\right)$ and the binning factor $\left(b_{y}\right)$ and is defined by

$1 / f_{\mathrm{b}}=t_{\exp }+t_{\mathrm{ro}} / b_{y}$.

The read-out time of the Imager QE is $100 \mathrm{~ms}$ when no binning is applied. The time for wavelength setting is below $0.5 \mathrm{~ms}$ and is thus negligible.

\subsection{New Fabry-Pérot etalons and controller}

Before describing the new FPI performance we mention that it has become possible to obtain from various manufacturers order sorting pre-filters with $F W H M$ of $6-10 \AA$ which have transmissions up to $80 \%$. Some 10 years ago filters were available with maximum transmissions of $40 \%$ and less. This improvement allows one to decrease the exposure time, or to decrease the noise level.

The formerly mounted high spectral resolution etalon from Burleigh Instruments with a diameter $\oslash=35 \mathrm{~mm}$ and a finesse $\mathcal{F} \leq 30$ has been replaced by an FPI (FPI1 in Fig. 1) from IC Optical Systems $(\oslash=70 \mathrm{~mm}, \mathcal{F} \sim 46$, spacing $1.1 \mathrm{~mm})$. The other FPI 2 from IC Optical Systems with $\oslash=50 \mathrm{~mm}, \mathcal{F} \sim 36$ and a spacing $0.125 \mathrm{~mm}$ has yet remained. It will be replaced by a second narrow-band etalon from IC Optical Systems with a diameter of also $70 \mathrm{~mm}$ in the future.

All etalons are controlled now by CS100 three-channel controllers of IC Optical Systems, with a three-axis capacitance bridge stabilisation system which enables the parallelism and cavity spacing of the etalon to be servo-stabilised. The CS100 allows to control the etalon spacing and parallelism to be better than $0.01 \%$ of a free spectral range (FSR). The controller also houses the power supplies to drive the etalon, along with front panel manual set-up and scan controls and rear panel interfaces for computer control. Drifts in mirror parallelism are avoided and wavelength drifts of maximum transmission are minimised by sealed reference capacitors. The spectrometer is mounted on a laboratory bench cast from stone with high heat capacity. It is operated in an air-conditioned laboratory and any residual drifts result from pressure changes of the air in the gap between the etalons. Long-duration tests of stability have yet to be performed (but see Fig. 7 below). Adjustment of the parallelism is done by applying an analogue sawtooth voltage to the piezos for the spacing. We then adjust the piezos for the parallelism, by controlling the output from a photomultiplier, such that the light from a He-Ne laser (6328 ̊) shows highest and narrowest emission. The spacing for scanning during observation is controlled digitally via an RS-232 port for scanning in wavelength.

\subsection{New software for data acquisition and FPI control}

Together with a new personal computer (PC), the Software Package Davis 7 from LaVision has been purchased, a powerful package for data acquisition and data analysis based on a C-like proprietary programming language called CL. To adapt the software to the spectrometer, new software with a Graphical User Interface (GUI) for camera read-out and FPI control has been developed and implemented. The FPI-GUI consists of 3 main parts, namely "hardware settings", "adjustment" and "observation".

The GUI part "hardware settings" allows for the selection of cameras, exposure time, frame rate, FOV and binning of the cameras. All parameters are stored in hardware setting files, that can be loaded individually.

The GUI part "adjustment" gives, on the one hand, the possibility of a continuous read-out of the cameras and allows for an overlay of the two images for a pixel precise alignment of the two cameras and to minimise rotation, displacement, and different image scale of the two images against each other, and thus to avoid the necessity of a post factum interpolation of the data sets to correct for these alignment errors. On the other hand the two etalons can be controlled individually or together for stepwise movement of their wavelength settings or scanning in wavelength. A line finding algorithm has been implemented which can be used for line positioning for the individual pre-filters in use.

In the GUI part "observation", finally, the selection of the main observing parameters is done: number of scans, cycle time and a scan table, where pre-filter, scan range and step width in wavelength as well as the number of images per wavelength position for each spectral range are given. Like in the case of the hardware settings all observing parameters are stored in individual user files which can be changed and loaded at any time and are handled automatically by the program. During the observations the pre-filter, image number, wavelength position, scan number, scan time, data processing time and total cycle time are displayed and all important observing parameters are stored additionally into a log-file. At the end of each scan the recorded images and the corresponding scanned mean line profile can be displayed. This option can be disabled to reduce the total cycle time and two allow fast time sequences.

\subsection{Spectral scanning}

The scanning of a spectral region is done as in most FPI spectrometers. The spacing of the etalons is changed, via computer control, to new wavelength positions. At each position several frames are taken for noise reduction during the image reconstruction process. It is now possible to observe several spectral regions quasi-simultaneously, by scanning one region shortly after the other, with a time delay of approximately $15 \mathrm{~s}$, with changes of pre-filters in between. For this purpose each spectral region obtains its own offset settings of the FPI spacing.

The spectral purity, i.e. the suppression of parasitic light from neighbouring orders of the FPIs and from the extended wings of the FPI transmission function, has improved somewhat for two reasons. Firstly, the finesse of the new 
narrow-band etalon with $\mathcal{F} \sim 46$ is substantially higher than that of the old one with $\mathcal{F} \leq 30$. This is partly due to an increased reflectivity of the coatings, but mainly to a better surface quality. Secondly, the spacing of this etalon is smaller than that of the former one, it is now $1.1 \mathrm{~mm}$ instead of the variable spacing of usually $1.5-2.0 \mathrm{~mm}$ of the formerly used etalon. In this way the $F W H M$ of approximately $40 \mathrm{~m} \AA$ has decreased only by a few $m \AA$. Yet the free spectral range FSR is now larger by a factor of 1.4-1.8, which decreases strongly the influence of the neighbouring orders which are not fully suppressed by the broadband etalon with a spacing of $0.125 \mathrm{~mm}$. The spectral resolution will substantially improve when a further narrow-band etalon is implemented as planned for the use of the spectrometer with the $1.5 \mathrm{~m}$ GREGOR solar telescope. This etalon will have a $70 \mathrm{~mm}$ aperture and $1.4 \mathrm{~mm}$ spacing, i.e. will be also narrow-band compared to the etalon with $0.125 \mathrm{~mm}$ spacing now in use. This in combination with the $1.1 \mathrm{~mm}$ etalon will strongly suppress both the side bands and the extended wings of the Airy transmission function for a single etalon.

The spurious light originating from multiple reflections back and forth between the two etalons (inter-etalon reflexes) is removed from the beam by slightly tilting the $0.125 \mathrm{~mm}$ (broadband) etalon about the longer side of the FOV such that the image from the first inter-etalon reflex is positioned just aside the desired image. The tilt angle amounts to $\alpha=\arctan [6.7 /(2 \times$ $1190)]=0.161$ (half of ratio of short chip dimension to focal length of camera lens). It causes a change of wavelength with maximum transmission of the broadband etalon across the FOV by $\Delta \lambda \sim-\left(\lambda_{0} / 2\right) \times(2 \alpha)^{2}=95 \mathrm{~m} \AA$ at $\lambda_{0}=6000 \AA$. If the tuning of the $1.1 \mathrm{~mm}$ (narrow-band) etalon is chosen properly the detuning of the two etalons against each other lies within the limits of $\pm 47.5 \mathrm{~m} \AA$. Such a detuning due to the tilting is unavoidable, in the present setup. It produces a progressive decrease of the total transmission and an increase of asymmetry of the total transmission profile towards the longer boundaries of the FOV.

These effects are small since the $0.125 \mathrm{~mm}$ etalon has a $F W H M \sim 0.4 \AA$ (at $6000 \AA$ ) which is large compared to the detuning. They are normally corrected during data analysis by means of flat-field profiles and line profiles, e.g., from the FTS Atlas (Brault \& Neckel, quoted by Neckel 1999). However, the detuning is of concern in connection with the unequal wavelength scanning discussed below.

With two narrow-band etalons in the collimated beam, as planned for the next step, the reflexes will be suppressed by placing an order sorting interference filter between the two etalons (see Kneer \& Hirzberger 2001 for a discussion of this problem). With the inter-etalon reflexes removed in this way, the two etalons need not be tilted against each other.

\section{Specific spectral properties}

In the former setup, the ratio of the spacings of the two etalons could be chosen as an integer number in the range of 8 to 16 . In the new system and in similar systems, this is not the case any more. The scanning step size in wavelength is a multiple of a minimum step given by the minimum change of physical spacing. In most cases, the minimum step widths in wavelength of the transmissions of the two FPIs are not integer multiples of each other. This inequality causes problems when scanning with both etalons, as described also by Tritschler et al. (2002). Therefore, one optimises the stepping to have the transmission maxima of the two etalons as close as possible. If, in addition, the etalons are not tuned, with their maxima of transmission not

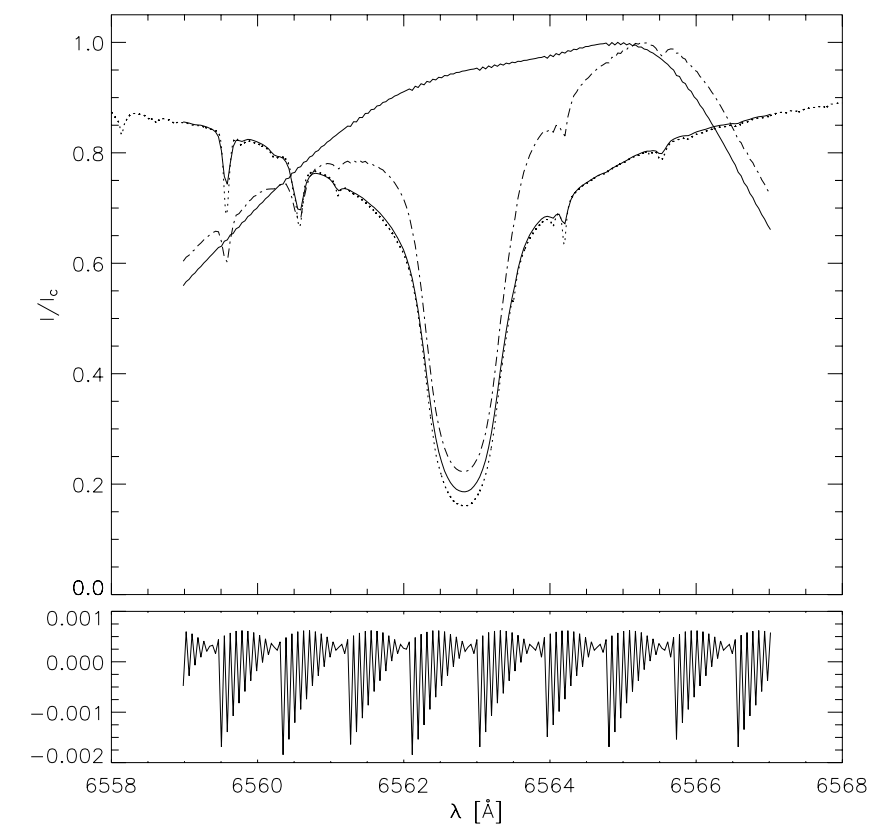

Fig. 3. Simulations of non-symmetric pre-filter, unequal wavelength scanning with the two etalons, and imperfect tuning. Upper panel: scanning through $\mathrm{H} \alpha$ with steps of $13 \times 3.09 \mathrm{~m} \AA$ and an imperfect tuning of $50 \mathrm{~m} \AA$. Dotted: $\mathrm{H} \alpha$ profile from the FTS Atlas (Brault \& Neckel, quoted by Neckel 1999). Thin solid: scan assuming only continuum, i.e. the transmission of an asymmetric pre-filter, normalised to its maximum intensity. Dash-dotted: "measured" $\mathrm{H} \alpha$ profile normalised to its maximum. Thick solid: measured $\mathrm{H} \alpha$ profile after division by the continuum profile and normalised to the maximum of the FTS profile. Lower panel: intensity fluctuations with the same scanning parameters as in the upper panel, but for pure continuum, in units of the average intensity.

coinciding, the inequality of wavelength steps gives noticeable changes of total transmission during scanning. With one transmission maximum in the flank of the transmission curve of the other, the unequal stepping results in discontinuities (jumps) in the measured intensity.

Figure 3 shows a simulation of unequal stepping with imperfect tuning, i.e. an offset of $50 \mathrm{~m} \AA$, included. As described above, such an offset is unavoidable with the etalons tilted against each other. The upper panel gives an example of a scan through the $\mathrm{H} \alpha$ line. The order sorting pre-filter has been assumed not fully symmetric, with somewhat higher transmission towards red. After correction for the inequality and the imperfect tuning by means of a "measurement" with a continuum light source, the final, "measured" profile possesses a somewhat smaller line depression as the profile from the Fourier Transform Spectrometer Atlas (Brault \& Neckel, quoted by Neckel 1999) due to the lower spectral resolution of our spectrometer, and due to the parasitic light from neighbouring orders of the narrowband FPI and to the extended wings of the transmission function. The lower panel of Fig. 3 depicts the relative intensity fluctuations without line and without pre-filter. We note that such fluctuations, and even much larger ones, have actually been seen in experiments in which we varied step width and added detuning on purpose. According to Tritschler et al. (2002) and to our experience, the resulting zig-zag pattern is very stable and can either be measured with a continuum lamp or be removed in the Fourier domain. The fluctuation amplitudes of $0.1-0.2 \%$ of the continuum intensity due to detuning of the FPI etalons are small. Yet they become much larger when both etalons have a very narrow transmission curve. Then, the locking 

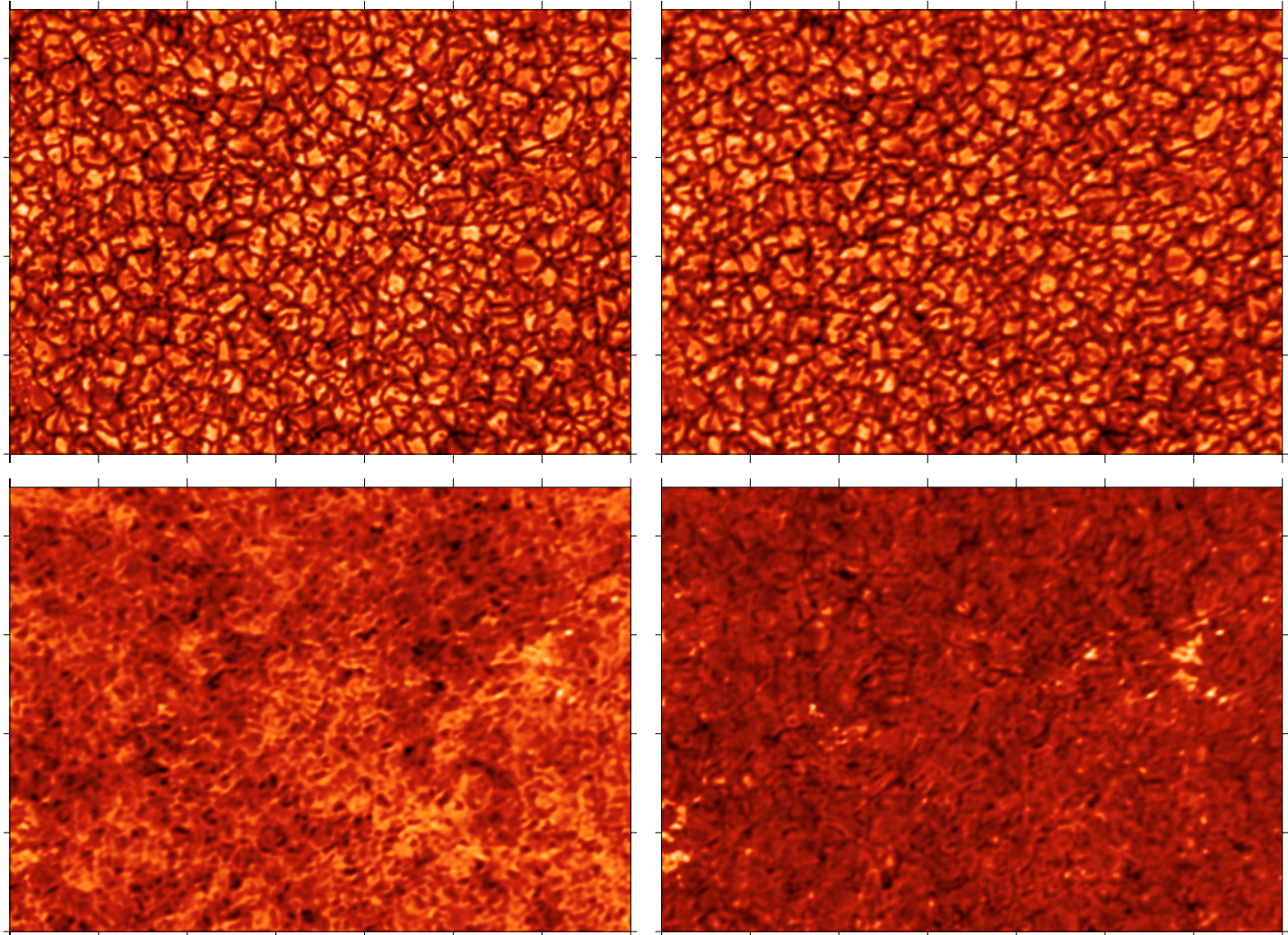

Fig. 4. First data from a wavelength scan in Fe I $6173 \AA$ A taken at disc centre of the Sun with the new system. The images are reconstructed with speckle methods. Shown are broadband image (upper left), image in continuum (upper right), image in the blue wing (lower left), and line centre image (lower right). Tick-marks at $10^{\prime \prime}$ distance. The FOV is $61.0^{\prime \prime} \times 43.6^{\prime \prime}$.

of several etalons, with their maxima of wavelength transmission coinciding as much as possible, has to be done very carefully. In addition, when small differences in intensity have to be measured, as in polarimetric observations, such intensity fluctuations may become of concern.

For the actual observations an algorithm is used to align the transmission maxima of the two etalons with an accuracy better than $5 \mathrm{~m} \AA$ for each wavelength range under study (but only for a small area in the FOV, since the tuning varies across the FOV). The resulting offsets are stored for each pre-filter and are used when spectral scans are performed.

\section{First observations}

First data with the FPI spectrometer were obtained on April 05, 2005 at the disk centre of the Sun. Narrow-band images $(F W H M=0.038 \AA)$ have been taken scanning the Fe I $6173 \AA$ line at 18 wavelength positions with 15 frames at each position. The step width in wavelength was $23.2 \mathrm{~m} \AA$. 270 broadband images $(F W H M=100 \AA)$ at $6302 \AA$ have been taken strictly simultaneously. The exposure times for both narrow-band and broadband CCDs were $3 \mathrm{~ms}$. Although this line is magnetically sensitive with a Landé factor $g=2.5$, polarimetric measurements were not performed for these first data.

The Adaptive Optics system of the Kiepenheuer Institute (KAOS, Soltau et al. 2002; von der Lühe et al. 2003) was used during the observations.

The broadband images were reconstructed with speckle methods applying the spectral ratio method by von der Lühe (1984) and the speckle masking by Weigelt (1977). With the estimate of the true broadband scenery on the Sun and with the instantaneous broadband images, the narrow-band images were reconstructed as described, e.g., by Krieg et al. (1999).

Figure 4 shows examples of the resulting images. The narrow-band continuum image exhibits almost the same sharpness as the speckle-reconstructed broadband image. The images in the wing and in the line centre show rich, detailed structuring. The improvement in sharpness of the narrow-band images was already mentioned above and noticed when test targets were placed in one focus in front of the spectrometer.

Since April 2005, some more data could be taken with the spectrometer, also polarimetric data. Quasi-simultaneous scans through the Fe I $6173 \AA$ and the $\mathrm{H} \alpha$ line have been performed. These data are under analysis and the results will be presented in forthcoming works. In Figs. 5 and 6 two examples of these observations are shown. Figure 5 gives results from a preliminary analysis of sunspot data near disc centre observed on 03 May, 2005 in Fe I $6173 \AA$ A. Figure 6 shows data of the solar south pole taken on 04 May, 2005 in $\mathrm{H} \alpha$. The observations have been performed with similar settings like the one stated above. For scanning the Fe I $6173 \AA$ line, a Stokes $V$ polarimeter has been used in front of the narrow-band CCD detector. The step width in $\mathrm{H} \alpha$ was $93 \mathrm{~mA}$. The integration time was $5 \mathrm{~ms}$ in both cases.

During all observational runs no drifts in wavelength have been observed like in case of the old system. The high and stable finesse of the new narrow-band etalon, the use of two CS-100 controllers and the digital etalon stepping control result in a high spectral stability of the wavelength scans performed. In Fig. 7 a comparison of the spectral stability of the old and the new system is presented. 28 profiles averaged over the FOV of 28 flat field scans, observed at consecutive time steps at Fe I $5434 \AA$ 

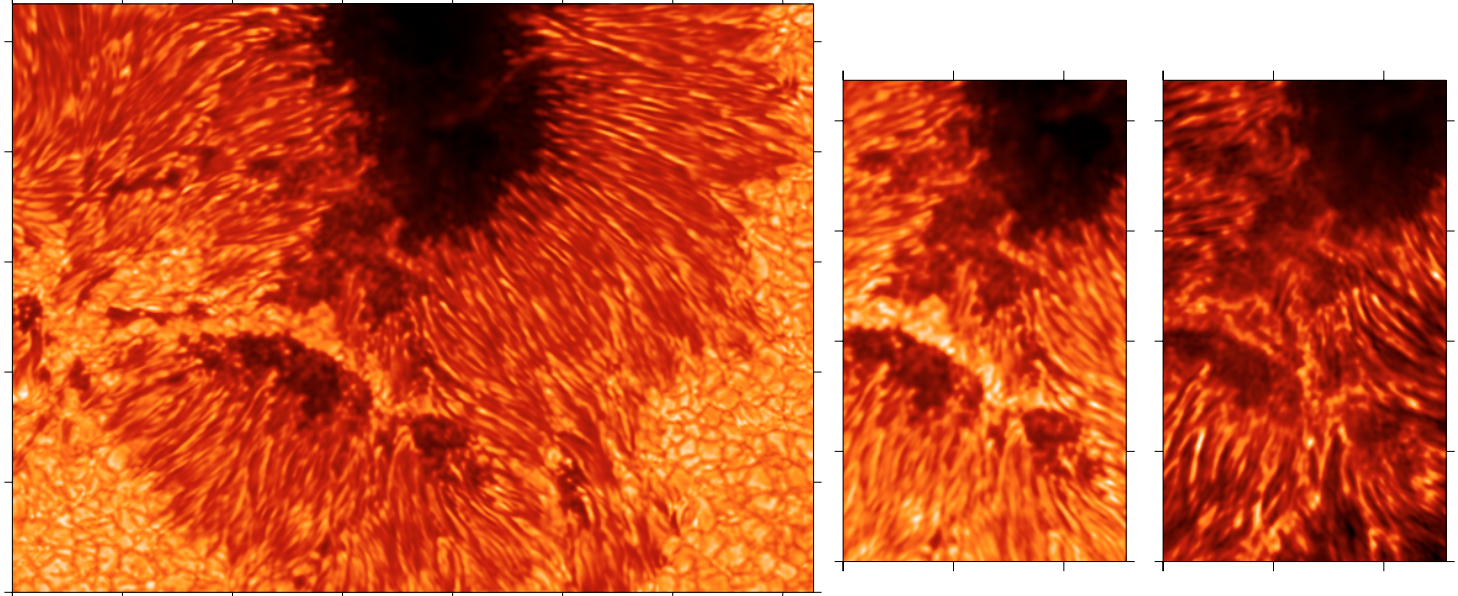

Fig. 5. Sunspot at $\vartheta=30^{\circ}$ in Fe I $6173.2 \AA$ observed on May 03, 2005. Left panel: speckle reconstructed broadband image $(F W H M=100 \AA)$; middle and right panels: reconstructed narrow-band $(F W H M=0.038 \AA$ ) Stokes $I-V$ images in the continuum and in the line centre, respectively. Tick-marks at $10^{\prime \prime}$ distance. The observations have been supported by Adaptive Optics.
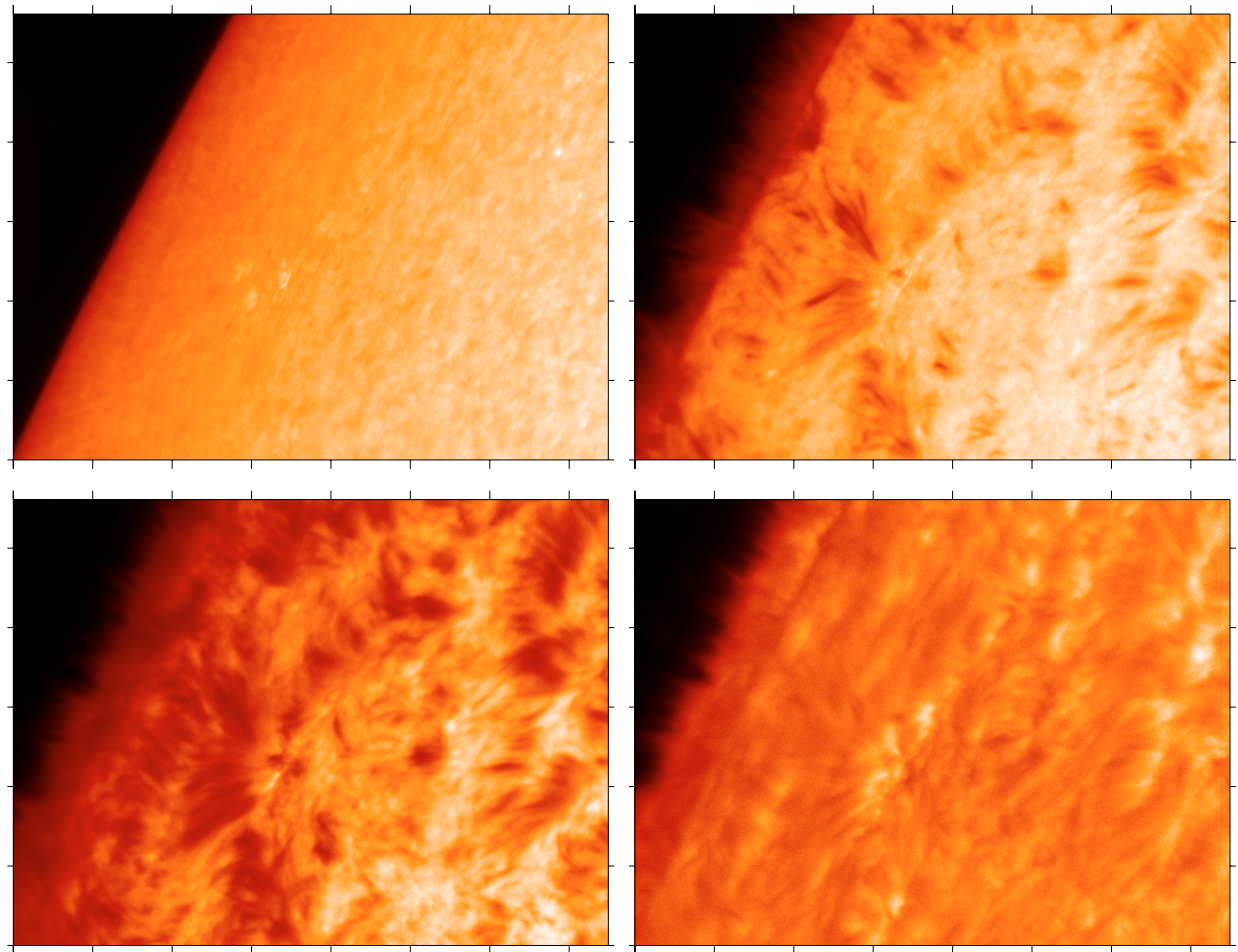

Fig. 6. Solar limb with spicules observed on 04 May, 2005. The observations have been supported by Adaptive Optics. Broadband image $(F W H M=$ $100 \AA$ ) at $6302 \AA$ (upper left). Narrow-band raw filtergrams $(F W H M=0.043 \AA)$ in H $\alpha$ at distances from line centre of $+0.651 \AA($ upper right), $+0.465 \AA$ (lower left), and line centre raw image (lower right). Tick-marks at $10^{\prime \prime}$ distance. The FOV is $77^{\prime \prime} \times 58^{\prime \prime}$.

and Fe I $6173 \AA$ on 16 April, 2003 and 23 August, 2005 are presented. The Fe I $5434 \AA$ line (Fe I $6173 \AA$ line) has been scanned, taking 4 (15) images at each 37 (22) spectral positions. The scan duration was $39 \mathrm{~s}(30 \mathrm{~s})$, the step width in wavelength $18.2 \mathrm{~m} \AA$ (23.2 $\mathrm{m} \AA)$ and the exposure time $20 \mathrm{~ms}(10 \mathrm{~ms})$. The total duration was $18.2 \mathrm{~min}(14.0 \mathrm{~min})$.

\section{Summary of performance}

Table 1 summarises the differences of performance between the former system and and the new spectrometer.

The exposure times have decreased to $3-5 \mathrm{~ms}$, while they were $20-30 \mathrm{~ms}$ with the old CCDs. The frame rate has increased from $3.5 \mathrm{~s}^{-1}$ to $18 \mathrm{~s}^{-1}$, i.e. by a factor of 5 . The FOV is now $77^{\prime \prime} \times 58^{\prime \prime}$ and has increased by a factor of 3.6 in area on the Sun.

We take the product of signal-to-noise ratio $(S N R)$, the frame rate $F R$, and FOV as a measure of the efficiency $E$ of the spectrometer,

$E \propto S N R \times F R \times F O V$.

$\operatorname{Be} \Phi_{\mathrm{ph}}$ the flux of photons (number of photons per ms and $\mu \mathrm{m}^{2}$ ) at the detector. The signal from the photons is then, in units of number of electrons,

$\Sigma_{\mathrm{ph}}=\Phi_{\mathrm{ph}} \cdot t_{\mathrm{exp}} \cdot F_{\mathrm{pix}} \cdot Q E$ 

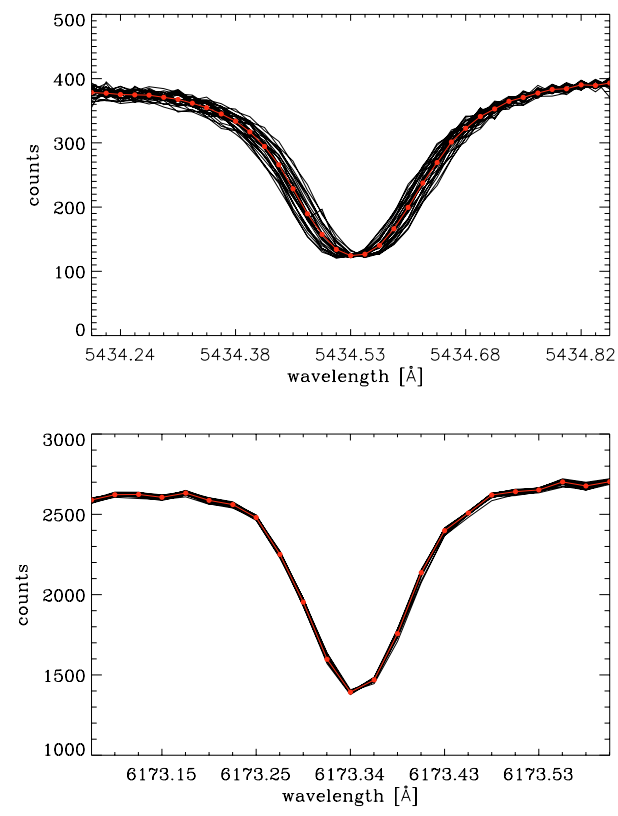

Fig. 7. Spectral stability. 28 profiles (black) averaged over the FOV of 28 flat field scans, observed at consecutive time steps at Fe I $5434 \AA$ (upper panel, old instrument) and Fe I $6173 \AA$ (lower panel, upgraded instrument). The Fe I $5434 \AA$ line (Fe I $6173 \AA$ line) has been scanned in $39 \mathrm{~s}(30 \mathrm{~s})$, the step width in wavelength was $18.2 \mathrm{~m} \AA$ ( $23.2 \mathrm{~m} \AA)$. The red profile (in the electronic version) in both panels corresponds to the temporal average. Red dots mark the scanned wavelength positions. The total duration was $18.2 \mathrm{~min}(14.0 \mathrm{~min})$.

with $t_{\exp }=$ exposure time, $F_{\text {pix }}=$ pixel area and $Q E=$ quantum efficiency. In units of counts, the signal is

$S_{\mathrm{ph}}=\Sigma_{\mathrm{ph}} / A D C$,

where $A D C=\mathrm{AD}$ conversion factor in $\mathrm{e}^{-} /$counts. The noise $N_{\mathrm{ph}}$ from the measured photons is in units of number of electrons

$N_{\mathrm{ph}}=\left(\Sigma_{\mathrm{ph}}\right)^{1 / 2}$.

The read-out noise $N_{\text {ro }}$ and the AD conversion noise $N_{\mathrm{AD}}$ have also to be taken into account to yield the total noise

$N_{\text {tot }}=\left(N_{\text {ph }}^{2}+N_{\text {ro }}^{2}+N_{\mathrm{AD}}^{2}\right)^{1 / 2}$.

As examples for measurement in a spectral line and in continuum, we take for the old system photon fluxes of 3 and 6 photons per ms and $\mu \mathrm{m}^{2}$, which gives the counts in typical measurements. For the new system, we assume the fluxes two times higher to account for the overall improved transmission after the upgrade. Exposure times of 20-30 ms with the former detectors were at the upper limit for reasonable speckle imaging. So, to calculate the $S N R$ of the old and new system, we adopt an exposure time of $t_{\exp }=3 \mathrm{~ms}$ and, in addition and for comparison of the $S N R, t_{\text {exp }}=30 \mathrm{~ms}$ for the old CCD. We take values for quantum efficiency, read-out noise, AD conversion noise, pixel area etc. from the data sheets of the CCDs and from Table 1.

For the pixel area of the new chip we assume $2 \times 2$ binning. To account for the different image scale (arcseconds per pixel) the pixel area on the old chip is taken as $F_{\text {pix }}=(12.9 \times$ $0.112 / 0.105 \mu \mathrm{m})^{2}$.

With Eqs. (3)-(6) and with the values given in Table 1 we arrive at the $S N R$ values in Table 2. Obviously, the new detectors compare favourably with the formerly used ones. With the short exposure times needed for speckle imaging $(\sim 3 \mathrm{~ms})$,
Table 1. Comparison of the performance of the upgraded spectrometer with the former system at $6173 \AA$ and for $2 \times 2$ binning of the new system.

\begin{tabular}{|c|c|c|}
\hline Properties & former & upgraded \\
\hline Narrow-band etalon & Burleigh Instruments: & IC Optical Systems Ltd.: \\
\hline Diameter [mm] & 35 & 70 \\
\hline Spacing [mm] & 1.5 & 1.1 \\
\hline Finesse & 30 & 46 \\
\hline Coating reflectivity [\%] & $94+2 /-4$ & $95 \pm 2$ \\
\hline Wavelength range [Å] & $4000-7800$ & $5300-8600$ \\
\hline Free spectral range $[\AA]$ & 1.27 & 1.73 \\
\hline FWHM Airy-funct. [̊̊] & 0.045 & 0.038 \\
\hline Spectral res. $[\lambda / \Delta \lambda]$ & 135000 & 160000 \\
\hline Narrow-band controller & Burleigh Instruments & IC Optical Systems Ltd. \\
\hline Wavelength scanning & analog & digital \\
\hline Wavelength drift & large & small \\
\hline Pre-filter transmission [\%] & $\sim 40$ & $\sim 80$ \\
\hline CCD & Thompson TH7863 FT & Sony, Imager QE \\
\hline Chip size $[\mathrm{mm}]$ & $8.8 \times 6.7$ & $8.8 \times 6.7$ \\
\hline $\begin{array}{l}\text { No. of pixels } \\
\text { (without binning) }\end{array}$ & $384 \times 286$ & $\begin{array}{r}688 \times 520 \\
1376 \times 1040\end{array}$ \\
\hline Pixel scale [arcsec] & 0.105 & 0.112 \\
\hline FOV [arcsec] & $40.3 \times 30.0$ & $77 \times 58$ \\
\hline Exp. times [ms] & $20-30$ & $3-5$ \\
\hline Frame rate $\left[\mathrm{s}^{-1}\right]$ & 3.5 & 18 \\
\hline Signal [counts] & $100-400(30 \mathrm{~ms})$ & $300-1500(3 \mathrm{~ms})$ \\
\hline $\mathrm{AD}$ conversion $\left[\mathrm{e}^{-} /\right.$count $]$ & $40-60$ & 2 \\
\hline $\mathrm{AD}$ conversion noise $\left[\mathrm{e}^{-}\right]$ & 25 & 1 \\
\hline Read-out noise $\left[\mathrm{e}^{-}\right]$ & 75 & 4.5 \\
\hline Dark signal $\left[\mathrm{e}^{-} / \mathrm{pix} / \mathrm{sec}\right]$ & 3000 at $\mathrm{T}=0^{\circ} \mathrm{C}$ & 1 at $\mathrm{T}=-12^{\circ} \mathrm{C}$ \\
\hline Dark non-uniformity [counts] & 50 & 1 \\
\hline Max. storage per scan & $\begin{array}{r}150 \text { frames } \\
(33 \mathrm{MB}) \text { into RAM }\end{array}$ & $\begin{array}{l}\text { up to } 600 \text { GB directly } \\
\text { onto hard disc }\end{array}$ \\
\hline
\end{tabular}

Table 2. Adopted photon fluxes $\Phi_{\mathrm{ph}}$, resulting signals $\Sigma_{\mathrm{ph}}$ and $S_{\mathrm{ph}}$, and signal-to-noise ratio $S N R$ at $6173 \AA$ for the former and the upgraded system for two different light levels. For the meaning of the other quantities see text. The numbers in brackets for the old system refer to an exposure time of $30 \mathrm{~ms}$.

\begin{tabular}{l|rr|rr}
\hline \hline & Former & & Upgraded & \\
\hline$\Phi_{\text {ph }}\left[\mathrm{ms}^{-1} \mu \mathrm{m}^{-2}\right]$ & 3 & 6 & 6 & 12 \\
$t_{\text {exp }}[\mathrm{ms}]$ & $3(30)$ & $3(30)$ & 3 & 3 \\
$F_{\text {pix }}\left[\mu \mathrm{m}^{2}\right]$ & & 189 & & 166 \\
$Q E$ & & 0.40 & & 0.53 \\
$A D C\left[\mathrm{e}^{-} /\right.$count $]$ & & 40 & & 2 \\
$\Sigma_{\mathrm{ph}}\left[\mathrm{e}^{-}\right]$ & $680(6800)$ & $1360(13600)$ & 1580 & 3170 \\
$S_{\mathrm{ph}}[$ counts $]$ & $17(170)$ & $34(340)$ & 790 & 1580 \\
$S N R$ & $8.2(60)$ & $16(96)$ & 40 & 56 \\
\hline
\end{tabular}

the $S N R$ has increased by a factor of 3.5 to 4.9 , depending on the light level. Only with exposure times of 20-30 ms formerly used CCDs have a SNR comparable with that of the new CCDs. Accordingly, using Eq. (2), the efficiency has increased by at least a factor of 60 . The upgraded system allows to perform 2D speckle spectroscopy and polarimetry in sunspot umbrae with exposure times of the order of $10 \mathrm{~ms}$, which were not feasible with the former system.

It is also worth noticing that, compared with the former spectrometer, the narrow-band images have become substantially sharper. Furthermore, the new system shows an improved spectral purity, i.e. a better suppression of parasitic light from neighbouring orders of the FPIs and from the extended wings of the FPI transmission function, due to the substantially higher finesse and the smaller spacing of the new narrow-band etalon, and a high spectral stability, resulting from the use of reference capacitors, the CS-100 etalon controllers and digital stepping control for both etalons. As a further extension of the scientific 
application, the feasibility of measuring quasi-simultaneously in several spectral regions has been added.

The system has new powerful hard- and software, the latter with a graphical user interface for image acquisition and FPI control. Test and optical adjustment procedures have been implemented and a comfortable visualisation of the data, of images and of line profiles is available. The data acquisition capability for one scan was formerly limited to 150 frames. Now, the data are directly stored onto a $600 \mathrm{~GB}$ raid disc of the new personal computer (PC), with data compression by a factor of 1.5.

\section{Conclusions and outlook}

The upgrade of the Göttingen two-dimensional spectrometer, accomplished during the first half of 2005, gave improvements in many respects. The substantial increase of efficiency by at least a factor of 60 reflects the rapid technical development, essentially on the side of the detectors and computers now available. The transmission and the sensitivity of the system increased substantially, the system has become much faster. Yet also the gain in stability of the optical components, mainly of the Fabry-Pérot etalons, has helped to meet today's and future requirements on high-resolution spectroscopy. Multi-line observations, i.e. scanning quasi-simultaneously through several spectral lines have been performed successfully in August 2005.

Most of these efforts have been undertaken in preparation of the $1.5 \mathrm{~m}$ GREGOR solar telescope (e.g., Volkmer et al. 2004). The Göttingen spectrometer will become a post-focus instrument for GREGOR. Then the low-resolution etalon with $0.125 \mathrm{~mm}$ spacing and free aperture of $50 \mathrm{~mm}$ will be replaced by a second narrow-band etalon with $70 \mathrm{~mm}$ free aperture and a $1.4 \mathrm{~mm}$ spacing. This in combination with the $1.1 \mathrm{~mm}$ etalon will strongly suppress both the side bands and the extended wings of the Airy transmission function. The implementation of a filterwheel into the narrow-band channel is in progress. It will allow the selection of up to 5 different narrow-band pre-filters for the quasi-simultaneous scanning of different spectral lines. Efforts are also undertaken to allow full Stokes polarimetry.
Acknowledgements. We thank the referee for careful reading and for constructive comments which helped to improve the quality of this publication. K.G.P. thanks the Deutsche Forschungsgemeinschaft for support through grant KN 152/29-1,2. Financial support by the German Bundesministerium für Forschung und Technologie through grant HBFG-103-589 is gratefully acknowledged. The Vacuum Tower Telescope is operated by the KiepenheuerInstitut für Sonnenphysik, Freiburg, at the Spanish Observatorio del Teide of the Instituto de Astrofísica de Canarias (Tenerife - Canary Islands).

\section{References}

Bello González, N., Okunev, O. V., Domínguez Cerdeña, I., Kneer, F., \& Puschmann, K. G. 2005, A\&A, 434, 317

Bendlin, C. 1993, Ph.D. Thesis, Göttingen university

Bendlin, C., \& Volkmer, R. 1993, A\&A, 278, 601

Bendlin, C., \& Volkmer, R. 1995, A\&AS, 112, 371

Bendlin, C., Volkmer, R., \& Kneer, F. 1992, A\&A, 257, 817

Bonaccini, D., Cavallini, F., Ceppatelli, G., \& Righini, A. 1989, A\&A, 217, 386

Bray, R. J. 1988, LEST Tech. Rep., 35, Inst. Theor. Astroph., Oslo university

Cavallini, F. 1998, A\&AS, 128, 589

Cavallini, F. 2002, in Proc. 2nd Solar Cycle and Space Weather Euroconference, ed. H. Sawaya-Lacoste, ESA-SP, 477, 585

Cavallini, F., Berrilli, F., Cantarano, S., \& Egidi, A. 2000, in Proc. 1st Solar \& Space Weather Euroconference, ed. A. Wilson, ESA-SP, 463, 607

Cavallini, F., Baffa, C., Reardon, K., et al. 2003, Mem. S. A. It., 74, 796

Janßen, K., Vögler, A., \& Kneer, F. 2003, A\&A, 409, 1127

Kentischer, T., Schmidt, W., Sigwarth, M., \& von Uexküll, M. 1998, A\&A, 340, 569

Kneer, F., \& Hirzberger, J. 2001, AN, 322, 375

Koschinsky, M., Kneer, F., \& Hirzberger, J. 2001, A\&A, 365, 588

Krieg, J., Wunnenberg, M., Kneer, F., Koschinsky, M., \& Ritter, C. 1999, A\&A, 343, 983

Loughhead, R. E., Bray, R. J., \& Brown, N. 1978, Appl. Opt., 17, 415

Martínez Pillet, V., et al. 2004, Optical, Infrared, and Millimeter Space Telescopes, Proc. SPIE, 5487, 1152

Neckel, H. 1999, Sol. Phys., 184, 421

Okunev, O. V., \& Kneer, F. 2004, A\&A, 425, 321

Ramsay, J. V., Kobler, H., \& Mugridge, E. G. V. 1970, Sol. Phys., 12, 492

Soltau, D., Berkefeld, T., von der Lühe, O., Wöger, F., \& Schelenz, T. 2002, AN, 323, 236

Tritschler, A., Schmidt, W., Langhans, K., \& Kentischer, T. 2002, Sol. Phys., 211, 17

Volkmer, R., Kneer, F., \& Bendlin, C. 1995, A\&A, 304, L1

Volkmer, R., von der Lühe, O., Kneer, F., et al. 2004, SPIE, 5489, 693

von der Lühe, O. 1984, J. Opt. Soc. Am. A1, 510

von der Lühe, O., \& Kentischer, T. 2000, A\&AS, 146, 499

von der Lühe, O., Soltau, D., Berkefeld, T., \& Schelenz, T. 2003, SPIE, 4853, 187

Weigelt, G. P. 1977, Opt. Comm., 21, 55

Wunnenberg, M., Kneer, F., \& Hirzberger, J. 2002, A\&A, 395, L51 